Aims. This Quality Improvement Project aimed to improve physical activity amongst patients in a 16-bedded, low secure unit in the Downshire Hospital, Northern Ireland. We introduced an exercise programme with the aim of increasing minutes of physical activity per week. Secondary outcome measures were weight, mood and energy levels.

This project took place in the context of COVID-19 restrictions having reduced opportunities for off-ward activity and staff noting subsequent deconditioning and weight gain amongst the patient cohort. Cohort consisted largely of patients with a severe mental illness, many of whom had physical health co-mobridities.

Method. This project included all patients in the 16-bedded unit.

Baseline data were collected prior to programme introduction, including weekly activity levels and weights. A questionnaire explored patient confidence and attitude towards physical activity.

Focus groups were held with patients and staff in order to identify how best to introduce the programme, discuss content, and identify potential barriers.

We introduced an eight-week programme of weekly, thirtyminute, mixed ability exercise sessions. These were led collectively by the multi-disciplinary team. Patients actively participated in programme design; choosing session soundtracks and contributing to content planning.

Likert scales were used to measure self-report mood and energy levels pre- and post-session. Staff engaged in a weekly post session de-brief, where challenges were identified and solutions suggested. Weekly qualitative feedback was sought from participants. The sessions were thus developed and adapted according to patient and staff feedback over the programme's course.

Following the 8-week programme, activity levels and weight were re-measured and compared to baseline. Pre-programme questionnaires were also repeated.

Result. Patients reported increased enjoyment and confidence engaging in physical activity, as well as improved overall self- confidence and a sense of pride and ownership of the sessions.

Staff reported a more cohesive team environment, greater sense of work-place fulfilment and improved therapeutic relationships.

Comparing pre and post session ten-point-Likert scales showed a $153 \%$ mean increase in self-rated energy levels and a $98 \%$ mean increase in self-rated mood. This reflected a mean score increase of 3.8 in both.

Minutes of physical activity per week increased for all session participants, although remained below national guidance.

Weight reduction did not occur.

Conclusion. Exercise benefits not only physical health, but also emotional and psychological well-being. This project demonstrates how introduction of a weekly ward-based exercise class can offer this as well as improving working environment, team cohesion and therapeutic relationships. Weight reduction may be observed in the longer term.

\section{Survey to evaluate care of complex clients in residential setting \\ Rosa Sadraei ${ }^{1 \star}$, Puru Pathy ${ }^{2}$ and Michael Collins ${ }^{1}$ \\ ${ }^{1}$ Nottinghamshire Healthcare NHS Foundation Trust and \\ ${ }^{2}$ Nottinghamshie Healthcare NHS Trust \\ ${ }^{\star}$ Corresponding author.}

doi: 10.1192/bjo.2021.577

Aims. Delivering a new efficient assessment and shorter term secondary mental Health intervention service for individual sectors
Background. In November 2015, there was a transition to services with the focus on delivering more efficient service to clients

Previously we had been a combined sector Service. This transition, a reduction in resources and a move away from delivering care Through specialist mental health teams created from the national service framework - such as Assertive outreach, early intervention in psychosis and community rehabilitation - to a more Streamlined generic service, catering for these differing groups of people using a "Pathways Model" approach

Result. Across the two sectors we had 47 clients on CPA Pathway living in 24 hour residential Settings who all had a current care coordinator.

These 47 clients represented the workload currently of 2.8 FTE Band 6 care coordinators.

There were at Origin, 13 Residential/Nursing/Secure 24 Hour care providers, where clients were residing.

However of these $90 \%$ of residents lived in one of 5 settings, 3 settings in Ashfield and 2 in Mansfield.

Over $50 \%$ of individuals residing did not have existing connections with Mansfield or Ashfield before being placed into the area.

18 Clients (\%38) were under section of the mental health act and 1 client (\%2) was on a life-Licence from criminal justice.

Conclusion. Transfer of CPA Care Coordination Protocol

To send paper referral to our Single Point of Access Meeting at the listed address at the earliest point relocation/placement is confirmed.Formal handover meeting for care will be coordinated, not sooner than 3 months after the placement commences. It will be expected that services currently involved in provision of service continue to hold care responsibility in the interim period.

As we move to a paperless environment, provision of electronic documentation such has previous CPA documents, Risk assessments, social circumstance reports \& Discharge summaries, would be greatly appreciated

\section{Patient \& staff perceptions of animal-assisted therapy in psychiatric rehabilitation}

Laura Sallette $^{1 \star}$, David King ${ }^{2}$, Sian Cowton-Williams ${ }^{1}$ and Rajesh Mohan ${ }^{1}$

${ }^{1}$ Heather Close Rehabilitation Unit, South London and Maudsley NHS Foundation trust and ${ }^{2}$ King's college london

${ }^{\star}$ Corresponding author.

doi: 10.1192/bjo.2021.578

Aims. To study patients' subjective experiences of having access to a therapy dog and to assess the staff perception of the impact of pet therapy. Hypothesis: pet therapy services are acceptable for rehabilitation patients.

Background. Animal-assisted therapy (AAT) is the supervised use of an animal in a therapeutic setting to help in the treatment of physical or psychological disorders in humans. The use of dogs in the context of AAT - 'dog therapy' (DT) has been piloted in the context of stroke rehabilitation; schizophrenia in elderly patients; depression, loneliness and anxiety in elderly patients; Alzheimer's disease; symptom reduction in PTSD; cognitive impairment; and dementia. The impact of pet therapy in long term psychosis care has not been adequately assessed.

Method. This feasibility pilot study used questionnaires to assess patient $(n=12)$ and staff $(n=10)$ perceptions of dog therapy in an in-patient psychiatric rehabilitation setting. 24 patients on a rehabilitation ward with complex psychosis were offered the opportunity to interact with 'Nugget,' a corgi trained in the 